\title{
Youth at Risk for Obesity Show Greater Activation of Striatal and Somatosensory Regions to Food
}

\author{
Eric Stice, ${ }^{1}$ Sonja Yokum, ${ }^{1}$ Kyle S. Burger, ${ }^{1}$ Leonard H. Epstein, ${ }^{2}$ and Dana M. Small 3,4 \\ ${ }^{1}$ Oregon Research Institute, Eugene, Oregon 97403, ${ }^{2}$ University at Buffalo, Behavioral Medicine, Buffalo NY, 14214, ${ }^{3}$ The John B. Pierce Laboratory, New \\ Haven, Connecticut 06519, and ${ }^{4}$ Yale University School of Medicine, Department of Psychiatry, New Haven, Connecticut 06511
}

\begin{abstract}
Obese humans, compared with normal-weight humans, have less striatal D2 receptors and striatal response to food intake; weaker striatal response to food predicts weight gain for individuals at genetic risk for reduced dopamine (DA) signaling, consistent with the rewarddeficit theory of obesity. Yet these may not be initial vulnerability factors, as overeating reduces D2 receptor density, D2 sensitivity, reward sensitivity, and striatal response to food. Obese humans also show greater striatal, amygdalar, orbitofrontal cortex, and somatosensory region response to food images than normal-weight humans do, which predicts weight gain for those not at genetic risk for compromised dopamine signaling, consonant with the reward-surfeit theory of obesity. However, after pairings of palatable food intake and predictive cues, DA signaling increases in response to the cues, implying that eating palatable food contributes to increased responsivity. Using fMRI, we tested whether normal-weight adolescents at high- versus low-risk for obesity showed aberrant activation of reward circuitry in response to receipt and anticipated receipt of palatable food and monetary reward. High-risk youth showed greater activation in the caudate, parietal operculum, and frontal operculum in response to food intake and in the caudate, putamen, insula, thalamus, and orbitofrontal cortex in response to monetary reward. No differences emerged in response to anticipated food or monetary reward. Data indicate that youth at risk for obesity show elevated reward circuitry responsivity in general, coupled with elevated somatosensory region responsivity to food, which may lead to overeating that produces blunted dopamine signaling and elevated responsivity to food cues.
\end{abstract}

\section{Introduction}

Overweight versus normal-weight rats have lower basal dopamine (DA) levels, D2 receptor availability, and induced DA release in the nucleus accumbens, dorsal striatum, and medial prefrontal cortex (Fetissov et al., 2002; Geiger et al., 2008). Obese versus normal-weight humans show less striatal D2 receptor availability (Volkow et al., 2008) and striatal response to food intake, which predicts future weight gain for individuals at genetic risk for compromised DA signaling (Stice et al., 2008a,b). These data are consistent with the reward-deficit theory, which posits that individuals who show hypo-responsivity of reward circuitry overeat or use psychoactive substances to compensate (Volkow et al., 2008).

Obese versus normal-weight humans also show greater caudate, gustatory region, and oral somatosensory region response to anticipated receipt of food (Stice et al., 2008b) and striatal, insular, orbitofrontal cortex (OFC), and amygdalar response to palatable food images, which predicts weight gain for those not at genetic risk for compromised DA signaling (Rothemund et al., 2007; Stoeckel et al., 2008; Stice et al., 2010a). These data are consistent with the reward-surfeit model of obesity, which holds that hyper-responsivity of reward circuitry leads to overeating and substance use (Dawe and Loxton, 2004).

\footnotetext{
Received Dec. 17, 2010; revised Jan. 11, 2011; accepted Jan. 24, 2011.

This work was supported by National Institutes of Health Grant DK-080760.

Correspondence should be addressed to Eric Stice, Oregon Research Institute, 1715 Franklin Boulevard, Eugene, Oregon 97403.E-mail: estice@ori.org.

DOI:10.1523/JNEUROSCI.6604-10.2011

Copyright $\odot 2011$ the authors $\quad 0270-6474 / 11 / 314360-07 \$ 15.00 / 0$
}

Findings suggest that obese humans show less activation of reward regions to food receipt, but greater activation in regions that encode the reward value of food cues. Yet, it is unclear whether the initial vulnerability for obesity is hypo-responsivity to food receipt or hyper-responsivity to food cues because overeating reduces D2 receptors density, D2 sensitivity, striatal response to food, and reward sensitivity (Kelley et al., 2003; Johnson and Kenny, 2010; Stice et al., 2010a) and repeated pairings of food intake and cues predicting food intake cause DA signaling increases in response to food cues (Kiyatkin and Gratton, 1994), suggesting that overeating leads to blunted striatal response to food and greater responsivity to food cues.

Findings are generally consistent with three competing working etiologic theories. One theory is that individuals at risk for obesity initially experience less reward from food intake, leading them to overeat to compensate for this reward deficit and to hyper-responsivity of reward circuitry via conditioning. A second theory is that individuals at risk initially show hyper-responsivity of regions that encode the reward value of food cues, leading to overeating and a reduction in DA signaling in response to food intake. A third theory is that individuals at risk initially experience greater reward from food intake, leading to overeating that reduces DA signaling in response to food intake and hyperresponsivity of reward circuitry to food cues, both of which may drive further overeating.

We tested these theories by comparing the activation of reward circuitry in response to receipt and anticipated receipt of food and monetary reward using functional magnetic resonance imaging in normal-weight youth at high-risk (HR) versus low- 
A

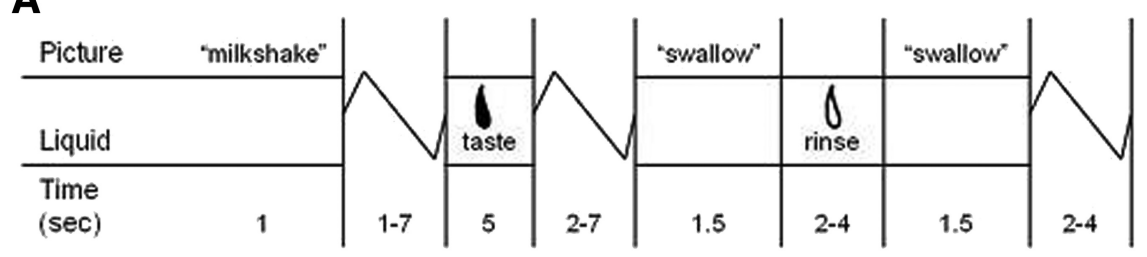

B

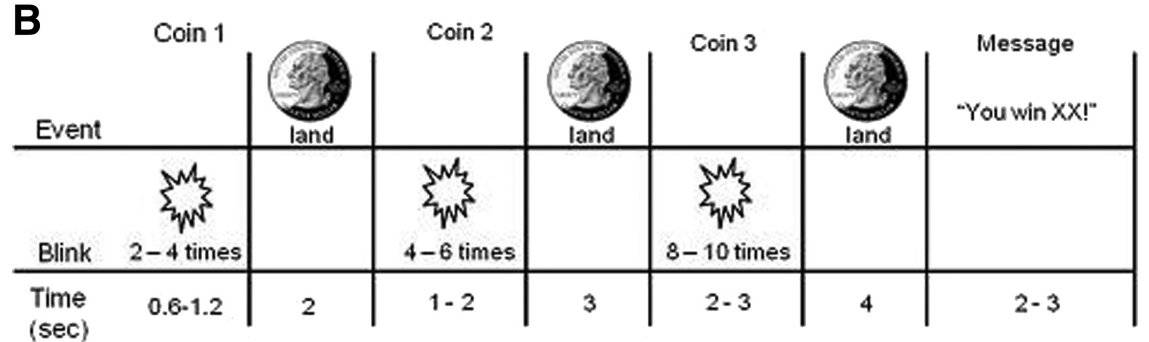

Figure 1. $A, B$, Examples of timing and ordering of presentation of pictures and drinks during the food reward paradigm $(A)$ and presentation of pictures during the monetary-reward paradigm $(\boldsymbol{B})$.

risk (LR) for obesity because there would be no possibility that a history of overeating contributed to anomalous responsivity. We investigated response to monetary reward to determine whether anomalies are specific to food or also apply to other rewards.

\section{Materials and Methods}

Participants. Participants were 30 female and 30 male lean adolescents (mean age, 15.0 years; SD, 2.9 years), of which 35 were high-risk children of two obese or overweight $(\mathrm{BMI} \geq 27)$ parents and 25 were low-risk children of two lean parents (BMI < 25) (where BMI is body mass index). Participants with two obese or overweight parents (mean parental $\left.\mathrm{BMI}_{\mathrm{HR}}=32.15 ; \mathrm{SD}=4.35\right)$ had a mean $\mathrm{BMI}_{\mathrm{HR}}$ of $20.64(\mathrm{SD}=1.67)$. Participants with two lean parents (mean parental $\mathrm{BMI}_{\mathrm{LR}}=23.13$; $\mathrm{SD}=$ 1.16) had a mean $\mathrm{BMI}_{\mathrm{LR}}$ of $20.07(\mathrm{SD}=1.80)$. Adolescent children of obese versus normal-weight parents show a fourfold increase in risk for obesity onset (Whitaker et al., 1997; Magarey et al., 2003). The sample consisted of 5\% Hispanic, 2\% Asian, 3\% African Americans, 85\% European Americans, and 5\% Native American participants. Parents provided written informed consent and adolescents provided written assent. Individuals who reported binge eating or compensatory behavior in the past 3 months, any use of psychotropic medications or illicit drugs, head injury with a loss of consciousness, or Axis I psychiatric disorder (including anorexia nervosa, bulimia nervosa, or binge eating disorder) in the past year were excluded.

fMRI paradigms. Participants were asked to consume their regular meals, but to refrain from eating or drinking (including caffeinated beverages) for 4-6 h immediately preceding their imaging session for standardization purposes. We selected this deprivation period to capture the hunger state that most individuals experience as they approach their next meal, which is a time when individual differences in reward circuitry responsivity would logically impact caloric intake. Because it was necessary to scan youth after school on weekdays, we were unable to feed participants a standardized meal $5 \mathrm{~h}$ before the scan. Participants were familiarized with the fMRI paradigms before the imaging session and the order of the presentation of the two paradigms (milkshake and money) was counterbalanced. Visual stimuli for both paradigms were presented with a digital projector/reverse screen display system to a screen at the back end of the MRI scanner bore and were visible via a mirror mounted on the head coil.

The food-reward paradigm (Fig. $1 A$ ) examines response to receipt and anticipated receipt of palatable food. Stimuli were presented in five separate, randomized scanning runs. The stimuli consisted of two images (glasses of milkshake and water) that signaled the delivery of either $0.5 \mathrm{ml}$ of a chocolate milkshake or a tasteless solution. Order of presentation was randomized. The milkshake consisted of 2 scoops of vanilla ice cream, 1.5 cups of $2 \%$ milk, and 2 tablespoons of chocolate syrup. The tasteless solution, designed to mimic the natural taste of saliva, consisted of $25 \mathrm{~mm} \mathrm{KCl}$ and $2.5 \mathrm{~mm} \mathrm{NaHCO}_{3}$ in distilled water (O’Doherty et al., 2001). We used artificial saliva because water has a taste that activates the taste cortex (Zald and Pardo, 2000). On $40 \%$ of the milkshake and tasteless solution trials, the taste was not delivered following the cue to allow the investigation of the neural response to anticipation of a taste that was not confounded with actual receipt of the taste (unpaired trials). There were six events of interest: (1) milkshake cue followed by milkshake receipt, (2) receipt of milkshake, (3) milkshake cue followed by no milkshake receipt, (4) tasteless solution cue followed by tasteless solution receipt, (5) receipt of tasteless solution, and (6) tasteless solution cue followed by no tasteless solution receipt. Cues were presented for $2 \mathrm{~s}$ and were followed by a jitter of 1-7 s during which time the screen was blank. Taste delivery occurred $\sim 10 \mathrm{~s}$ after cue onset and lasted $5 \mathrm{~s}$. The trial ended with a second jitter of 2-7 s. Each event lasted between 2 and $5 \mathrm{~s}$. Tastes were delivered using programmable syringe pumps to ensure consistent volume, rate, and timing of taste delivery. Sixty milliliters syringes filled with milkshake and tasteless solution were connected via Tygon tubing through a wave guide to a manifold attached to the sliding table of the scanner. The manifold fit into the participants' mouths and delivered the taste to a consistent segment of the tongue. This procedure has been successfully used previously (Stice et al., 2008a,b, 2010b). Participants were instructed to swallow when they saw the "swallow" cue. The next cue appeared 2-4 s after the "swallow" cue went off. Participants completed a cross-modal visual analog scale to rate the perceived pleasantness, edibility, and intensity of the milkshake and tasteless solution before the scan.

The monetary reward paradigm (Fig. $1 B$ ) was developed to assess activation in response to receipt and anticipated receipt of monetary reward, based on the monetary incentive delay paradigm (Knutson et al., $2001 \mathrm{a}, \mathrm{b})$. We selected monetary reward because it is a general reinforcer, has been frequently used in behavioral and fMRI paradigms assessing reward sensitivity, and has been used as a standard of comparison for different types of reward (Izuma et al., 2008; Rademacher et al., 2010). Stimuli, consisting of three coins (heads or tails), were presented in two separate runs. Order of presentation of the runs was randomized. During the run, a coin on the left-hand side of the screen would blink two to four times (during blinking, the stimulus was presented for $300 \mathrm{~ms}$ ) and would then stay on the screen. After $2 \mathrm{~s}$, a second coin would blink four to six times before remaining in the middle of the screen. After $3 \mathrm{~s}$, a third coin would blink eight to 10 times and then remain on the screen for $4 \mathrm{~s}$. After the presentation of the coins, a 2-3 s message appeared, saying whether or not the subject has won ("You win \$3" or "You don't win"). Stimulus presentations were jittered. The subject won $\$ 3$ each time three heads or three tails were displayed. During each run, the total amount earned was presented below the coins ("Your total is $\$ X X ")$. There were three events of interest: (1) winning $\$ 3$, indicated by three matching coins (all heads or all tails); (2) a potential win, indicated by two matching coins (two heads or two tails in a row); and (3) a reward-neutral coin display, indicated by the first coin (one head or one tail). In total, there were 10 repeats of the experimental event of interest (five repeats of three heads plus five repeats of three tails) and 16 repeats of the other events.

$f M R I$ data acquisition, preprocessing, and statistical analysis. Scanning was performed by a Siemens Allegra 3 tesla head-only MRI scanner. A birdcage coil acquired data from the entire brain. A thermo-foam vacuum pillow and additional padding restricted head motion. Functional scans used a T2*-weighted gradient single-shot echo planar imaging sequence [echo time, $30 \mathrm{~ms}$; repetition time (TR), $2000 \mathrm{~ms}$; flip angle, $80^{\circ}$ ] with an in-plane resolution of $3.0 \times 3.0 \mathrm{~mm}^{2}[64 \times 64$ matrix; field of 

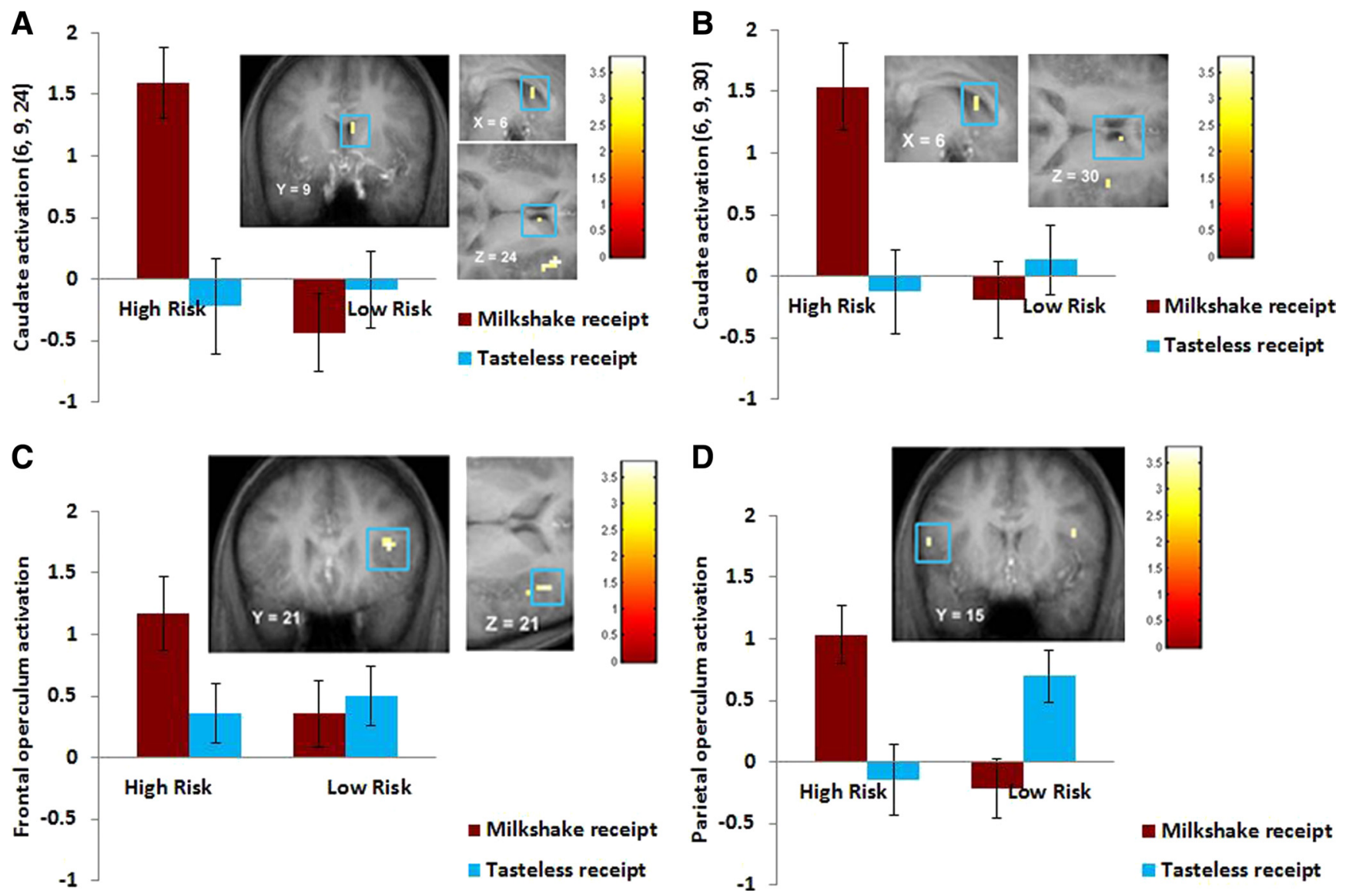

Figure 2. $A-D$, Greater activation in the right caudate $(A: 6,9,24 ; Z=3.14 ; p=0.04, F D R ; k=3 ; B: 6,9,30 ; Z=3.23, p=0.04, F D ; k=3)$, right frontal operculum $(\boldsymbol{C}: 39,21,21 ; Z=3.44$, $p=0.02, \mathrm{FDR} ; k=5)$, and left parietal operculum $(\boldsymbol{D}:-54,15,21 ; Z=3.36 ; p=0.02, \mathrm{FDR} ; k=2)$ in the high-risk versus low-risk group during milkshake receipt-tasteless receipt, with the bar graphs of parameter estimates from those peak voxels.

view (FOV), $192 \times 192 \mathrm{~mm}^{2]}$. To cover the whole brain, $324 \mathrm{~mm}$ slices (interleaved acquisition, no skip) were acquired along the anterior commissure-posterior commissure transverse, oblique plane, as determined by the midsagittal section. Structural scans were collected using an inversion recovery T1-weighted sequence (MP-RAGE) in the same orientation as the functional sequences to provide detailed anatomic images aligned to the functional scans. High-resolution structural MRI sequences (FOV, $256 \times 256 \mathrm{~mm}^{2} ; 256 \times 256$ matrix; thickness, $1.0 \mathrm{~mm}$; slice number $\approx 160$ ) were acquired.

Data were preprocessed and analyzed using SPM5 (Wellcome Department of Imaging Neuroscience, London, UK) in MATLAB (MathWorks) (Worsley and Friston, 1995). Images were time-acquisition corrected to the slice obtained at $50 \%$ of the TR. Functional images were then realigned to the mean. Images (anatomical and functional) were normalized to the standard Montreal Neurological Institute (MNI) template brain implemented in SPM5 (ICBM152, based on an average of 152 normal MRI scans). Normalization resulted in a voxel size of $3 \mathrm{~mm}^{3}$ for functional images and a voxel size of $1 \mathrm{~mm}^{3}$ for structural images. Functional images were smoothed with a $6 \mathrm{~mm}$ full-width at half maximum isotropic Gaussian kernel.

To identify brain regions activated in response to food receipt, we contrasted blood oxygenation level-dependent (BOLD) activation during receipt of milkshake versus receipt of tasteless solution. To identify regions activated in response to anticipated food receipt, BOLD activation during presentation of the cue signaling impending delivery of the milkshake was contrasted with response during presentation of the cue signaling impending delivery of the tasteless solution. We analyzed data from trials in which the tastes were not actually delivered to ensure that taste receipt would not influence our operationalization of anticipatory brain activation. To identify regions activated in response to receipt of monetary reward, we contrasted BOLD activation at the time a participant won (third coin stopped blinking and matched previous two) versus a reward-neutral coin display (the time the first coin stopped blinking, which conveyed no information about possible monetary reward). The neutral comparison was used because of evidence that losing in monetary paradigms activates similar areas to winning money (Knutson et al., 2001a; Elliott et al., 2003; Small et al., 2005). To identify regions activated in response to anticipation of monetary receipt, BOLD activation during presentation of the cue signaling a potential win (i.e., two heads or two tails in a row) was contrasted with the reward-neutral coin display (i.e., one head or one tail). Condition-specific effects at each voxel were estimated using general linear models. Vectors of the onsets for each event of interest were compiled and entered into the design matrix so that eventrelated responses could be modeled by the canonical hemodynamic response function, as implemented in SPM5, consisting of a mixture of two gamma functions that emulate the early peak at $5 \mathrm{~s}$ and the subsequent undershoot.

To account for variance induced by swallowing the solutions during the food reward paradigm, we included the time of the swallow cue as a covariate. We also included temporal derivatives of the hemodynamic function to obtain a better model of the data (Henson et al., 2002). A $128 \mathrm{~s}$ high-pass filter (per SPM5 convention) was used to remove lowfrequency noise and slow drifts in the signal.

Individual maps were constructed to compare the activations within each participant for the contrasts: milkshake receipt-tasteless receipt, milkshake cue-tasteless cue, time of win-reward-neutral coin display, and time of a potential win-reward-neutral coin display. Between-group comparisons were then performed using random-effect models to account for interparticipant variability. For the food reward paradigm parameter estimate, images were entered into a mixed between- and 

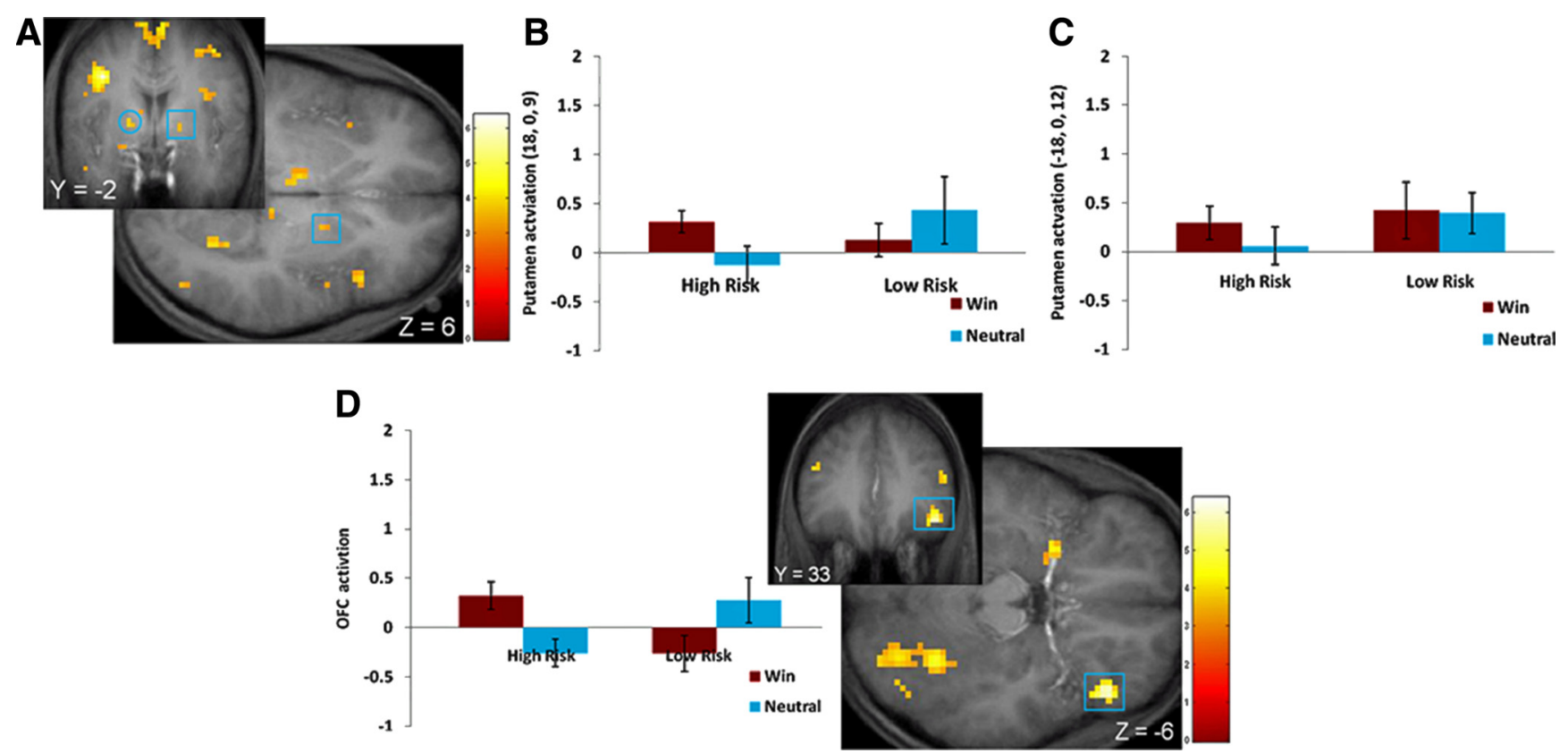

Figure 3. $A-D$, Greater activation in high-risk versus low-risk group in response to the win-neutral display in the right putamen $(\boldsymbol{A}, \mathrm{square}: 18,0,9 ; Z=3.44 ; p=0.018, \mathrm{FDR} ; \mathrm{k}=3)$, left putamen (circle: $-18,0,12 ; Z=3.74, p=0.007$, FDR, whole brain; $k=5$ ) with bar graphs of the parameter estimates from those regions ( $\boldsymbol{B}$, right putamen; $\boldsymbol{C}$, left putamen) and in the right orbitofrontal cortex ( $\boldsymbol{D}: 45,33,-6 ; Z=5.61, p<0.001, \mathrm{FDR} ; k=16)$ with bar graphs of the parameter estimates from that voxel.

within-subjects second-level $2 \times 2$ ANOVA [(high-risk vs low-risk) by (milkshake receipt-tasteless receipt or unpaired milkshake cue-unpaired tasteless cue)]. For analysis of monetary reward paradigm parameter estimate, images were entered into a second level mixed between and within-subjects $2 \times 2$ ANOVA [(high-risk vs low-risk) by (win-neutral coin display or potential win-neutral coin display)].

We performed small volume correction analyses on regions of interest in areas associated with food and monetary reward (caudate, putamen, insula, orbitofrontal cortex, and thalamus), somatosensory regions (operculuar regions), and attention (visual cortex). A priori-defined small volumes were based on activation peaks found in previous fMRI studies with similar food (Stice et al., 2008a,b) and monetary reward (Knutson et al., 2001b; Elliott et al., 2003; Small et al., 2005; Rademacher et al., 2010) paradigms as centroids to define $10 \mathrm{~mm}$ diameter spheres. T-map threshold was set at $p=0.001$ uncorrected and a three-voxel cluster size. Predicted activations were considered to be significant at $p<$ 0.05 after correcting for multiple comparisons $[p$, false discover rate (FDR)] across the voxels within the a priori-defined small volumes. Peaks outside these regions were considered to be significant at $p<0.05$ FDR, corrected across the whole brain. All stereotactic coordinates are presented in MNI space (http://mni.mcgill.ca/).

\section{Results}

There was no significant difference between high-risk (mean $=$ 13.89; $\mathrm{SD}=1.47$ ) and low-risk (mean $=14.5$; $\mathrm{SD}=2.02$ ) participants in pleasantness ratings of the milkshake $\left(t_{(58)}=-1.40\right.$, $p=0.17)$. High-risk versus low-risk participants showed greater activation in the right caudate $\left(\eta^{2}=0.11\right.$ and 0.16$)($ Fig. $2 A, B)$, right frontal operculum $\left(\eta^{2}=0.12\right)$ (Fig. $\left.2 C\right)$, and left parietal operculum $\left(\eta^{2}=0.15\right)$ (Fig. $\left.2 D\right)$ during milkshake receipttasteless solution receipt. No significant differences emerged in response to the unpaired cue predicting impending chocolate milkshake receipt-the unpaired cue predicting impending tasteless solution receipt. When we conducted the reverse low-risk versus high-risk contrast, there were no significant differences in activation during the milkshake receipt-tasteless solution receipt contrast or the unpaired cue predicting milkshake-unpaired cue predicting tasteless solution contrast.
Table 1. Greater regional brain activity in high-risk for obesity relative to low-risk participants in response to monetary reward (win-neutral contrast)

\begin{tabular}{|c|c|c|c|c|c|c|c|}
\hline & $x^{a}$ & $y$ & $Z$ & $k^{b}$ & $Z$ value & $p$, FDR corrected $^{c}$ & $\eta^{2 d}$ \\
\hline \multicolumn{8}{|c|}{ Anterior insula } \\
\hline \multirow[t]{4}{*}{$\mathrm{R}$} & 33 & 18 & 12 & 23 & 3.69 & 0.000 & 0.10 \\
\hline & 42 & 18 & 9 & & 3.64 & 0.000 & \\
\hline & 39 & 18 & 18 & & 3.42 & 0.000 & \\
\hline & 30 & 24 & 12 & & 3.38 & 0.000 & \\
\hline $\mathrm{L}$ & -36 & 18 & 15 & 50 & 4.34 & 0.000 & 0.08 \\
\hline \multicolumn{8}{|c|}{ Thalamus } \\
\hline \multirow[t]{2}{*}{$\mathrm{R}$} & 12 & -9 & 18 & 17 & 3.90 & 0.005 & 0.07 \\
\hline & 6 & -6 & 21 & & 3.75 & 0.005 & \\
\hline \multirow[t]{2}{*}{ L } & -9 & -15 & 3 & 18 & 4.10 & 0.000 & 0.05 \\
\hline & -6 & -15 & 9 & & 3.99 & 0.000 & \\
\hline \multicolumn{8}{|c|}{ Visual cortex } \\
\hline \multirow[t]{3}{*}{$\mathrm{R}$} & 27 & -78 & -6 & 35 & 4.00 & 0.000 & 0.09 \\
\hline & 27 & -78 & -6 & & 3.76 & 0.000 & \\
\hline & 39 & -81 & -3 & & 3.34 & 0.000 & \\
\hline \multirow[t]{2}{*}{ L } & -24 & -93 & 24 & 38 & 4.20 & 0.000 & 0.05 \\
\hline & -27 & -87 & 33 & & 3.94 & 0.000 & \\
\hline \multicolumn{8}{|c|}{ Orbitofrontal cortex } \\
\hline $\mathrm{R}$ & 45 & 33 & -6 & 16 & 5.61 & 0.000 & 0.13 \\
\hline \multicolumn{8}{|c|}{ Caudate } \\
\hline L & -12 & 3 & 18 & 3 & 3.25 & 0.013 & 0.09 \\
\hline \multicolumn{8}{|c|}{ Putamen } \\
\hline$R$ & 18 & 0 & 9 & 3 & 3.44 & 0.018 & 0.06 \\
\hline L & -18 & 0 & 12 & 5 & 3.74 & $0.007^{e}$ & 0.08 \\
\hline
\end{tabular}

R, Right; L, left.

${ }^{a}$ Stereotactic coordinates in MNI space.

${ }^{b}$ Cluster size.

'T maps thresholded at $p<0.001$ (uncorrected) with a cluster threshold of three. Peaks were considered significant at $p<0.05$, FDR corrected across the small volume.

${ }^{d}$ Effect size.

eFDR corrected across the whole brain.

High-risk versus low-risk participants showed greater activation in the right putamen $\left(\eta^{2}=0.06\right)$ (Fig. $\left.3 A, B\right)$, left putamen $\left(\eta^{2}=0.08\right)$ (Fig. $\left.3 A, C\right)$, right OFC $\left(\eta^{2}=0.13\right)$ (Fig. $\left.3 D\right)$, and left caudate boundary (Table 1 ) in response to winning money-the 
Table 2. Within-group comparisons for the low-risk group contrasting differences in the food-reward paradigm and the monetary-reward paradigm

\begin{tabular}{|c|c|c|c|}
\hline Contrast and region & $k$ & Zvalue & MNI coordinates \\
\hline \multicolumn{4}{|l|}{ Milkshake receipt $>$ tasteless solution receipt } \\
\hline \multirow[t]{2}{*}{ Superior frontal gyrus } & 707 & 5.00 & $21,-12,63$ \\
\hline & 251 & 4.74 & $-21,-15,75$ \\
\hline \multirow[t]{2}{*}{ Parietal lobe } & 427 & 4.91 & $-33,-60,30$ \\
\hline & 49 & 3.99 & $36,-60,27$ \\
\hline \multirow[t]{2}{*}{ Putamen } & 43 & 4.68 & $21,-3,30$ \\
\hline & 39 & 3.12 & $-24,3,21$ \\
\hline \multirow[t]{2}{*}{ Cerebellum } & 84 & 4.62 & $-21,-33,-18$ \\
\hline & 121 & 4.31 & $18,-36,-24$ \\
\hline Middle temporal gyrus & 10 & 4.42 & $-51,0,-24$ \\
\hline Medial prefrontal cortex/orbitofrontal cortex & 21 & 4.09 & $-6,36,-6$ \\
\hline Anterior cingulate cortex & 21 & 3.88 & $-12,27,0$ \\
\hline Midbrain & 13 & 3.85 & $-3,-30,-24$ \\
\hline Amygdala & 9 & 3.78 & $-27,-15,-3$ \\
\hline Medial frontal gyrus & 25 & 3.74 & $-6,-24,60$ \\
\hline Cingulate gyrus & 11 & 3.73 & $-9,-45,30$ \\
\hline Inferior frontal gyrus & 5 & 3.69 & $-48,30,0$ \\
\hline Inferior parietal lobe & 16 & 3.60 & $-39,-42,51$ \\
\hline Inferior temporal gyrus & 3 & 3.54 & $63,-9,-18$ \\
\hline Caudate & 4 & 3.41 & $-6,12,18$ \\
\hline \multicolumn{4}{|l|}{ Unpaired milkshake cue $>$ unpaired tasteless cue } \\
\hline \multirow[t]{2}{*}{ Occipital lobe } & 122 & 4.69 & $-12,-90,3$ \\
\hline & 40 & 3.72 & $18,-69,9$ \\
\hline \multirow[t]{2}{*}{ Cerebellum } & 18 & 4.07 & $-27,-60,-30$ \\
\hline & 6 & 3.45 & $33,-45,-30$ \\
\hline \multicolumn{4}{|l|}{ Winning $>$ neutral } \\
\hline Parietal lobe & 85 & 4.01 & $9,-81,48$ \\
\hline Occipital lobe & 26 & 3.74 & $-12,-72,12$ \\
\hline Anterior cingulate cortex & 3 & 3.28 & $0,24,33$ \\
\hline \multicolumn{4}{|l|}{ Impending win > neutral } \\
\hline Thalamus & 7 & 3.78 & $0,6,21$ \\
\hline Middle frontal gyrus & 10 & 3.63 & $-36,6,39$ \\
\hline Middle occipital gyrus & 8 & 3.52 & $-24,-69,6$ \\
\hline Rolandic operculum & 7 & 3.55 & $-36,-24,36$ \\
\hline Mid insula & 3 & 3.47 & $-36,9,24$ \\
\hline Frontal operculum & 3 & 3.30 & $-51,6,12$ \\
\hline
\end{tabular}

For all contrasts, activated regions, number of contiguous voxels $(k), Z$ values, and coordinates of the voxel of greatest activation within the MNI coordinate system are displayed. Peaks within the regions were considered significant at $p<0.001$, uncorrected for multiple comparisons.

reward-neutral display. Significant activation also emerged in the bilateral anterior insula, bilateral thalamus, and bilateral visual cortex in response to monetary reward (Table 1). No significant differences emerged in response to anticipation of winning money-the reward-neutral display or for the reverse contrasts during receipt or anticipated receipt of monetary reward.

\section{Discussion}

The finding that normal-weight adolescents at high-risk versus low-risk for future obesity showed greater activation in the dorsal striatum in response to palatable food receipt is novel. This suggests that the initial vulnerability that gives rise to obesity may be elevated rather than blunted sensitivity of the dorsal striatum to food reward. It is thus possible that lower dorsal striatal response to food receipt (Stice et al., 2008a,b) and reduced D2 receptor availability (Wang et al., 2001; Volkow et al., 2008) for obese versus normal-weight individuals is a consequence rather than a cause of overeating. This possibility aligns with evidence that overeating leads to downregulation of postsynaptic D2 receptors, decreased D2 sensitivity, and reduced reward sensitivity in rodents (Colantuoni et al., 2001; Bello et al., 2002; Kelley et al., 2003; Johnson and Kenny, 2010) and that weight gain results in reduced striatal response to palatable food intake in humans (Stice
Table 3. Within-group comparisons for the high-risk group contrasting differences in the food reward paradigm and the monetary reward paradigm

\begin{tabular}{lrrc}
\hline Contrast and region & $k$ & Z value & MNI coordinates \\
\hline Milkshake receipt $>$ tasteless solution receipt & & & \\
Superior parietal lobe & 766 & 4.41 & $12,-42,78$ \\
Amygdala & 66 & 3.80 & $-27,-3,-15$ \\
& 7 & 3.80 & $30,-3,-9$ \\
Postcentral gyrus & 743 & 4.63 & $-42,-33,63$ \\
Rolandic operculum & 177 & 4.48 & $42,-33,45$ \\
Temporal operculum & 96 & 4.28 & $-57,-15,9$ \\
Caudate & 17 & 4.20 & $6,12,24$ \\
Frontal operculum & 11 & 3.85 & $48,6,21$ \\
& 9 & 3.80 & $-45,3,21$ \\
Inferior parietal lobe & 36 & 3.80 & $27,-54,39$ \\
Putamen & 12 & 3.70 & $-27,3,12$ \\
Dorsolateral prefrontal cortex & 5 & 3.51 & $39,42,50$ \\
Thalamus, ventral lateral nucleus & 4 & 3.37 & $15,-15,6$ \\
Medial orbitofrontal cortex & 3 & 3.15 & $33,36,-6$ \\
Unpaired milkshake cue $>$ unpaired tasteless cue & & & \\
Occipital lobe & 74 & 4.82 & $-12,90,0$ \\
& 32 & 3.82 & $33,-69,-3$ \\
Cuneus & 56 & 3.82 & $21,-90,3$ \\
Posterior cingulate gyrus & 25 & 3.59 & $-6,-57,24$ \\
Winning $>$ neutral & & & \\
Posterior cingulate gyrus extending into occipital lobe & 1289 & 6.14 & $18,-63,12$ \\
Anterior cingulate cortex & 18 & 4.22 & $0,12,42$ \\
Precentral gyrus & 5 & 3.91 & $51,-9,57$ \\
Superior temporal gyrus & 3 & 3.55 & $-60,-57,15$ \\
Impending win $>$ neutral & & & \\
Occipital lobe & 264 & 5.15 & $-3,-75,9$ \\
Superior temporal gyrus & 4 & 3.43 & $-60,-57,15$ \\
\hline
\end{tabular}

For all contrasts, activated regions, number of contiguous voxels $(k), Z$ values, and coordinates of the voxel of greatest activation within the MNI coordinate system are displayed. Peaks within the regions were considered significant at $p<0.001$, uncorrected for multiple comparisons.

et al., 2010a). It is important to note that some of the significant interactions conform to the expected pattern of effects better than others. The findings shown in Figure 2, $A-C$, indicate that high-risk youth showed greater activation in reward regions in response to milkshake receipt relative to tasteless solution receipt compared with low-risk youth. However, the interaction shown in Figure $2 D$ emerged in part because the activation pattern was reversed for the low-risk relative to the high-risk group, even though the parietal operculum activation was still greater for high-risk versus low-risk youth. This is because the group-byreward receipt interactions expressly identified peaks for which the activation pattern for reward receipt (vs the contrast condition) differed for high-risk versus low-risk groups. In this context, it is also important to note that post hoc analyses confirmed that both the low-risk and the high-risk youth showed significantly greater activation of reward circuitry in response to receipt of milkshake versus tasteless solution, including the caudate, putamen, amygdala, and medial orbitofrontal cortex (Tables 2, 3), partially validating the paradigm.

The finding that youth at high-risk versus low-risk for future obesity showed greater striatal response to monetary reward is also novel. This suggests that the initial vulnerability that increases risk for obesity may be a general hyper-responsivity of the dorsal striatum to various reward types, rather than specific to food reward, with this hyper-responsivity applying to both primary (food) and conditioned (money) rewards. However, it is important to note that the interactions shown in Figure 3 did not conform to the expected pattern as well as the food reward interactions; although the high-risk youth did show greater activation in response to winning money then the control contrast, each of 
these interactions were driven in part by the fact that the activation pattern was qualitatively different for the low-risk youth, such as Figure $3 D$. Thus, the conclusion that high-risk youth showed greater reward circuitry responsivity to monetary reward should be interpreted in this light. Nonetheless, post hoc analyses confirmed that both the low-risk and the high-risk youth showed greater activation of reward circuitry in response to winning money versus the contrast condition (e.g., in the anterior cingulate cortex) (Tables 2, 3).

As would be expected, the elevated oral somatosensory response was only observed in response to food and not in response to monetary reward. This finding extends evidence that obese versus normal-weight individuals show greater activation of oral somatosensory regions in response to anticipated palatable food receipt (Stice et al., 2008b) and images of palatable foods (Stice et al., 2010a), greater regional blood flow in somatosensory regions in response to images of palatable foods (Karhunen et al., 1997), and greater resting glucose metabolism in the oral somatosensory cortex (Wang et al., 2002). Moreover, since fat is detected primarily by the oral somatosensory system via cues about creaminess, viscosity, and texture (de Araujo and Rolls, 2004), these findings also accord with evidence that obese versus normal-weight humans rate high-fat foods as more pleasant (Drewnowski et al., 1985; McGloin et al., 2002; Rissanen et al., 2002), that children at risk for obesity by virtue of parental obesity prefer the taste of high-fat foods relative to children of normal-weight parents (Fisher and Birch, 1995; Stunkard et al., 1999; Wardle et al., 2001), and that preferences for high-fat foods predict elevated future weight gain (Stunkard et al., 1999; Salbe et al., 2004). This suggests that individuals at risk for obesity exhibit a hyperresponsivity to reward in general, but that this may need to be coupled with a hyper-responsivity of somatosensory regions to convey specific risk for obesity versus other appetitive behavioral problems (e.g., drug abuse, gambling, compulsive sexuality).

We did not observe significant differential response to anticipation of either food or monetary reward as a function of obesity risk, suggesting that elevated sensitivity of regions that encode the reward value of food cues may not be an initial vulnerability factor for obesity. The evidence that conditioning leads to an increased responsiveness of reward circuitry to food cues (Schultz et al., 1993; Kiyatkin and Gratton, 1994) putatively via a learning mechanism implies that recurrent overeating may produce the elevated activation of the dorsal striatum and other reward regions in response to images of palatable foods and anticipated food receipt observed in obese versus normal-weight individuals (Rothemund et al., 2007; Stice et al., 2008b, 2010a; Stoeckel et al., 2008).

Collectively, extant findings suggest the possibility of a dynamic vulnerability model for obesity that may evolve and change over time in response to overeating. Data suggest that individuals at risk for obesity initially show hyper-responsivity of the striatum to reward in general and somatosensory regions in response to palatable, energy-dense foods, which may increase risk for overeating. We posit that the oral somatosensory responses reflect altered sensitivity for fat and/or enhanced preference for fat foods. We further submit that overeating may in turn result in a downregulation of DA-based reward regions, producing a blunted striatal response to food intake, which may lead people to overeating in an effort to achieve the same subjective reward from palatable food they initially experienced (i.e., chasing the high) in a feed-forward manner. The overeating may also result in greater responsivity of regions that encode the reward value of food cues, making people more vulnerable to food cues in our obesogenic environment, which also may increase risk for escalation of overeating in a feed-forward fashion.

\section{References}

Bello NT, Lucas LR, Hajnal A (2002) Repeated sucrose access influences dopamine D2 receptor density in the striatum. Neuroreport 13:1575-1578.

Colantuoni C, Schwenker J, McCarthy J, Rada P, Ladenheim B, Cadet JL, Schwartz GJ, Moran TH, Hoebel BG (2001) Excessive sugar intake alters binding to dopamine and mu-opioid receptors in the brain. Neuroreport 12:3549-3552.

Dawe S, Loxton NJ (2004) The role of impulsivity in the development of substance use and eating disorders. Neurosci Biobehav Rev 28:343-351.

de Araujo IE, Rolls ET (2004) Representation in the human brain of food texture and oral fat. J Neurosci 24:3086-3093.

Drewnowski A, Brunzell JD, Sande K, Iverius PH, Greenwood MR (1985) Sweet tooth reconsidered-taste responsiveness in human obesity. Physiol Behav 35:617-622.

Elliott R, Newman JL, Longe OA, Deakin JF (2003) Differential response patterns in the striatum and orbitofrontal cortex to financial reward in humans: a parametric functional magnetic resonance imaging study. J Neurosci 23:303-307.

Fetissov SO, Meguid MM, Sato T, Zhang LH (2002) Expression of dopaminergic receptors in the hypothalamus of lean and obese Zucker rats and food intake. Am J Physiol Regul Integr Comp Physiol 283:R905-R910.

Fisher JO, Birch LL (1995) Fat preferences and fat consumption of 3-yearold to 5-year-old children are related to parental adiposity. J Am Diet Assoc 95:759-764.

Geiger BM, Behr GG, Frank LE, Caldera-Siu AD, Beinfeld MC, Kokkotou EG, Pothos EN (2008) Evidence for defective mesolimbic dopamine exocytosis in obesity-prone rats. FASEB J 22:2740-2746.

Henson RN, Price CJ, Rugg MD, Turner R, Friston KJ (2002) Detecting latency differences in event-related BOLD responses: application to words versus nonwords and initial versus repeated face presentations. Neuroimage 15:83-97.

Izuma K, Saito DN, Sadato N (2008) Processing of social and monetary rewards in the human striatum. Neuron 58:284-294.

Johnson PM, Kenny PJ (2010) Dopamine D2 receptors in addiction-like reward dysfunction and compulsive eating in obese rats. Nat Neurosci 13:635-641.

Karhunen LJ, Lappalainen RI, Tammela L, Turpeinen AK, Uusitupa MI (1997) Subjective and physiological cephalic phase responses to food in obese binge-eating women. Int J Eat Disord 21:321-328.

Kelley AE, Will MJ, Steininger TL, Zhang M, Haber SN (2003) Restricted daily consumption of a highly palatable food (chocolate Ensure(R)) alters striatal enkephalin gene expression. Eur J Neurosci 18:2592-2598.

Kiyatkin EA, Gratton A (1994) Electrochemical monitoring of extracellular dopamine in nucleus accumbens of rats lever-pressing for food. Brain Res 652:225-234.

Knutson B, Adams CM, Fong GW, Hommer D (2001a) Anticipation of increasing monetary reward selectively recruits nucleus accumbens. J Neurosci 21:RC159.

Knutson B, Fong GW, Adams CM, Varner JL, Hommer D (2001b) Dissociation of reward anticipation and outcome with event-related fMRI. Neuroreport 12:3683-3687.

Magarey AM, Daniels LA, Boulton TJ, Cockington RA (2003) Predicting obesity in early adulthood from childhood and parental obesity. Int J Obes Relat Metab Disord 27:505-513.

McGloin AF, Livingstone MB, Greene LC, Webb SE, Gibson JM, Jebb SA, Cole TJ, Coward WA, Wright A, Prentice AM (2002) Energy and fat intake in obese children and lean children at varying risk of obesity. Int J Obes Relat Metab Disord 26:200-207.

O’Doherty J, Rolls ET, Francis S, Bowtell R, McGlone F (2001) Representation of pleasant and aversive taste in the human brain. J Neurophysiol 85:1315-1321.

Rademacher L, Krach S, Kohls G, Irmak A, Gründer G, Spreckelmeyer KN (2010) Dissociation of neural networks for anticipation and consumption of monetary and social rewards. Neuroimage 49:3276-3285.

Rissanen A, Hakala P, Lissner L, Mattlar CE, Koskenvuo M, Rönnemaa T (2002) Acquired preference especially for dietary fat and obesity: a study of weight-discordant monozygotic twin pairs. Int J Obes Relat Metab Disord 26:973-977. 
Rothemund Y, Preuschhof C, Bohner G, Bauknecht HC, Klingebiel R, Flor H, Klapp BF (2007) Differential activation of the dorsal striatum by highcalorie visual food stimuli in obese individuals. Neuroimage 37:410 -421.

Salbe AD, DelParigi A, Pratley RE, Drewnowski A, Tataranni PA (2004) Taste preferences and body weight changes in an obesity-prone population. Am J Clin Nutr 79:372-378.

Schultz W, Apicella P, Ljungberg T (1993) Responses of monkey dopamine neurons to rewarded and conditioned stimuli during successive steps of learning a delayed response task. J Neurosci 13:900-913.

Small DM, Gitelman D, Simmons K, Bloise SM, Parrish T, Mesulam MM (2005) Monetary incentives enhance processing in brain regions mediating top-down control of attention. Cereb Cortex 15:1855-1865.

Stice E, Spoor S, Bohon C, Small DM (2008a) Relation between obesity and blunted striatal response to food is moderated by TaqIA Al allele. Science 322:449-452.

Stice E, Spoor S, Bohon C, Veldhuizen MG, Small DM (2008b) Relation of reward from food intake and anticipated food intake to obesity: a functional magnetic resonance imaging study. J Abnorm Psychol 117:924-935.

Stice E, Yokum S, Bohon C, Marti N, Smolen A (2010a) Reward circuitry responsivity to food predicts future increases in body mass: moderating effects of DRD2 and DRD4. Neuroimage 50:1618-1625.

Stice E, Yokum S, Blum K, Bohon C (2010b) Weight gain is associated with reduced striatal response to palatable food. J Neurosci 30:13105-13109.

Stoeckel LE, Weller RE, Cook EW 3rd, Twieg DB, Knowlton RC, Cox JE
(2008) Widespread reward-system activation in obese women in response to pictures of high-calorie foods. Neuroimage 41:636-647.

Stunkard AJ, Berkowitz RI, Stallings VA, Schoeller DA (1999) Energy intake, not energy output, is a determinant of body size in infants. Am J Clin Nutr 69:524-530.

Volkow ND, Wang GJ, Telang F, Fowler JS, Thanos PK, Logan J, Alexoff D, Ding YS, Wong C, Ma Y, Pradhan K (2008) Low dopamine striatal D2 receptors are associated with prefrontal metabolism in obese subjects: possible contributing factors. Neuroimage 42:1537-1543.

Wang GJ, Volkow ND, Logan J, Pappas NR, Wong CT, Zhu W, Netusil N, Fowler JS (2001) Brain dopamine and obesity. Lancet 357:354-357.

Wang GJ, Volkow ND, Fowler JS (2002) The role of dopamine in motivation for food in humans: implications for obesity. Expert Opin Ther Targets 6:601-609.

Wardle J, Guthrie C, Sanderson S, Birch L, Plomin R (2001) Food and activity preferences in children of lean and obese parents. Int J Obes Relat Metab Disord 25:971-977.

Whitaker RC, Wright JA, Pepe MS, Seidel KD, Dietz WH (1997) Predicting obesity in young adulthood from childhood and parental obesity. N Engl J Med 337:869-873.

Worsley KJ, Friston KJ (1995) Analysis of fMRI time-series revisited-again. Neuroimage 2:173-181.

Zald DH, Pardo JV (2000) Cortical activation induced by intraoral stimulation with water in humans. Chem Senses 25:267-275. 